\title{
A novel spectral formulation for transversely isotropic magneto-elasticity
}

2017, Vol. 22(5) 1158-1176

(C) The Author(s) 2015

Reprints and permissions:

sagepub.co.uk/journalsPermissions.nav

DOI: $10.1177 / 1081286515618999$

journals.sagepub.com $/$ home $/ \mathrm{mms}$

(SSAGE

\author{
M.H.B.M. Shariff \\ Department of Applied Mathematics and Science, Khalifa University of Science, Technology and Research, \\ United Arab Emirates
}

\section{Roger Bustamante}

Departamento de Ingenieria Mecanica, Universidad de Chile, Santiago, Chile

\section{Mokarram Hossain}

Chair of Applied Mechanics, University of Erlangen-Nuremberg, Erlangen, Germany

Mechanical and Construction Engineering, Northumbria University, Newcastle upon Tyne, UK

\author{
Paul Steinmann \\ Chair of Applied Mechanics, University of Erlangen-Nuremberg, Erlangen, Germany
}

Received 9 March 2015; accepted I November 2015

\begin{abstract}
Classical invariants, despite most of them having unclear physical interpretation and not having experimental advantages, have been extensively used in modeling nonlinear magneto-elastic materials. In this paper, a new set of spectral invariants, which have some advantages over classical invariants, is proposed to model the behavior of transversely isotropic nonlinear magneto-elastic bodies. The novel spectral invariant formulation, which is shown to be more general, is used to analytically solve some simple magneto-mechanical boundary value problems. With the aid of the proposed spectral invariants it is possible to study, in a much simpler manner, the effect of different types of deformations on the response of the magneto-elastic material.
\end{abstract}

\section{Keywords}

Magneto-elasticity, magneto-mechanical coupled problem, nonlinear elasticity, spectral invariants

\section{Introduction}

In the last few years there has been a growing interest in the study of the interactions of large elastic or viscoelastic deformations with magnetic fields [1-20]. One of the applications of such theories is the modeling of the behavior of magneto-active elastomers [6-10, 12, 16-19] (see also [11, 21-35]). These works are based on classical treatises developed in the past, for example, the works by Brown [36], Eringen and Maugin [37] and Maugin [38] among others (see also [39]). Magneto-active elastomers can display relatively large deformations 
if an external magnetic field is applied [11,21-35]. The possibility of controlling such deformation by tuning the external magnetic field justifies the classification as smart materials. Magneto-active elastomers have been proposed to be used in robotic and vibration control applications [27, 40-49].

One example of this class of elastomers corresponds to the case of a rubber-like matrix material filled with magneto-active particles $[6,21,22,24,31,35,43]$. When the particles are randomly distributed inside the body, we speak about an isotropic magneto-active or magneto-sensitive (MS) elastomer. When an external magnetic field is applied, isotropic MS elastomers behave in a similar manner as transversely isotropic solids where the preferred direction is induced by the magnetic field and the corresponding magnetic forces.

During the curing process, it is possible to apply an external magnetic field, which produces a relative alignment of the magneto-active particles that remain locked within chains when the body solidifies $[6,22,31$, $35,43,50-52]$. This class of magneto-active elastomers is called transversely isotropic MS elastomers [2], and it has been shown that the magnetostriction effect in such materials is much stronger in comparison to the case of isotropic MS elastomers [6, 22, 31, 35, 43, 50,53]. Therefore, such materials are more interesting from the point of view of possible applications. In the case of a transversely MS elastomer, when an external magnetic field is applied, the material behaves as a solid with two families of fibers, where one preferred direction is given by the magneto-active particle chains, whereas the additional preferred direction is induced by the magnetic fields.

In this work we are interested in studying the behavior of transversely isotropic MS elastomers, based on the formulation of Dorfmann and Ogden [7-10], where the main concept is the use of a total energy function, which depends on the deformation gradient and one of the independent magnetic variables, such as the magnetic induction. It has been shown that the total stress tensor and the magnetic field can be expressed as simple derivatives of the total energy function in terms of the deformation gradient and the magnetic induction [810]. If a body has two preferred directions, the representation theory of Spencer and Rivlin (see [54] and also [55]) indicates that the energy function should depend on ten invariants in terms of the right CauchyGreen deformation tensor and the magnetic induction in the reference configuration (see, for example, [2]). The expressions for the total stress tensor and the magnetic field obtained using such a classical set of invariants can be complicated and long (see, for example, $[2,17]$ ). Therefore, when proposing specific expressions for the total energy function from experimental data, it is necessary to consider a few simplifications concerning such an energy function, assuming, for example, that such a function depends only on a subset of the set of invariants [2]. Recently Shariff [56-59] has proposed a different method to find invariants for the energy function, for the purely elastic case, when considering transversely isotropic bodies [56] and also bodies with two preferred directions [59]. The main idea is to construct a set of spectral (Lagrangian axes) invariants whose elements are the principal stretches and the square of the dot product of the eigen directions of the right stretch tensor with the preferred directions. These spectral invariants have clear physical meanings which in turn give clear expressions for the stress components in the sense that it is easier to separate and to analyze the effect of considering the different invariants and the effects of the different kinds of deformations on the stress components.

Considering the above remarks, in this communication a set of spectral invariants is presented in order to model the behavior of transversely isotropic MS bodies as a natural extension of the analysis presented for isotropic MS bodies [60]. This new set is based on the spectral invariants considered by Shariff and Bustamante [59] for purely elastic deformations in two preferred direction materials. The new invariants have clear physical meanings, thus they can be more attractive in order to look for concrete expressions for the total energy function by fitting experimental data, and also in order to design a rational program of experiments for MS elastomers. In addition to this, classical invariants can be explicitly expressed in terms of the spectral invariants, and hence if the constitutive equation is initially written in terms of classical invariants, the relevant formulations can be easily formulated in terms of either classical or spectral invariants. However, if for the onset the constitutive equation is written in terms of spectral invariants it is generally impossible to convert it explicitly to classical invariants and we cannot write the relevant formulations in terms of the classical invariants. Hence, expressing the energy function in terms of the spectral invariants is more general. A numerical procedure, such as the finite element method, can be easily constructed based on the spectral formulations developed in this paper.

This paper is divided into 6 sections. In Section 2 some fundamental relations for the kinematics of the deformation of a body are presented, and some of the basic expressions of the theory of Dorfmann and Ogden [21] for nonlinear MS solids are revisited. In Section 3 the new set of invariants for transversely isotropic MS bodies is presented, and the expressions for the total stresses and the magnetic variable are derived. In Section 4, some simple boundary value problems are solved using the new set of invariants. Before we give final remarks in Section 6, we make some comments on a specific constitutive equation in Section 5. 


\section{Basic equations}

\section{I. Kinematics}

In this paper subscripts $i, j, k$ take the values $1,2,3$, unless stated otherwise. Let $\mathscr{B}$ denote the MS body and $\boldsymbol{x} \in \mathcal{B}_{t}$ denote the position of a particle $X \in \mathscr{B}$ in the current configuration $\mathcal{B}_{t}$. The position of the same particle in the reference configuration is denoted as $\boldsymbol{X} \in \mathcal{B}_{0}$, where $\mathcal{B}_{0}$ is the body in the reference configuration, which we assume is undeformed and unstressed. It is assumed that there exists a one-to-one mapping $\boldsymbol{x}=\chi(\boldsymbol{X}, t)$ for any time $t>0$. The deformation gradient and the left and right Cauchy-Green tensors, $\boldsymbol{b}$ and $\boldsymbol{C}$, are defined, respectively, as

$$
\boldsymbol{F}=\frac{\partial \boldsymbol{x}}{\partial \boldsymbol{X}}, \quad \boldsymbol{b}=\boldsymbol{F} \boldsymbol{F}^{\mathrm{T}}=\boldsymbol{V}^{2}, \quad \boldsymbol{C}=\boldsymbol{F}^{\mathrm{T}} \boldsymbol{F}=\boldsymbol{U}^{2},
$$

where we assume $\chi$ is such that $J=\operatorname{det} \boldsymbol{F}>0,\{\bullet\}^{\mathrm{T}}$ is the transpose of a second order tensor, and $\boldsymbol{V}$ and $\boldsymbol{U}$ are the left and right stretch tensors, respectively. We have used a lower case character to denote the left Cauchy-Green deformation tensor, in order to avoid problems with the notation used in magnetostatics. More details about kinematics can be found, for example, in [61, 62]. In this work we only consider quasi-static deformations.

\subsection{Magnetostatics}

In the following sections we review briefly some elements of the theory of magnetostatics [63] and of the theory for nonlinear MS bodies by Dorfmann and Ogden [9]. Let $\boldsymbol{H}$ and $\boldsymbol{B}$ denote the spatial magnetic field and the spatial magnetic induction, respectively, i.e. in the current configuration. In the absence of electric interactions and time effects, the magnetic field and the magnetic induction have to satisfy the simplified form of the Maxwell equations

$$
\operatorname{div} \boldsymbol{B}=0, \quad \operatorname{curl} \boldsymbol{H}=\mathbf{0} .
$$

Using the global form of (2), it is possible to define the following Lagrangian counterparts (i.e. in the reference configuration) of the magnetic field $\boldsymbol{H}_{l}$ and the magnetic induction $\boldsymbol{B}_{l}$ (see, for example, [7-9]):

$$
\boldsymbol{H}_{l}=\boldsymbol{F}^{\mathrm{T}} \boldsymbol{H}, \quad \boldsymbol{B}_{l}=J \boldsymbol{F}^{-1} \boldsymbol{B} .
$$

In a vacuum the magnetic field and the magnetic induction are related by the constitutive equation

$$
\boldsymbol{B}=\mu_{0} \boldsymbol{H},
$$

where $\mu_{0}$ is the magnetic permeability in a vacuum. In condensed matter an additional field is required, i.e. the magnetization $\boldsymbol{M}$, which is related to $\boldsymbol{B}$ and $\boldsymbol{H}$ through

$$
\boldsymbol{B}=\mu_{0}[\boldsymbol{H}+\boldsymbol{M}]
$$

\subsection{Magneto-elasticity}

The presence of a magnetic field in an MS body creates magnetic body forces and torques. As a result the mechanical stress tensor is not symmetric [38]. One of the key points of the theory of Dorfmann and Ogden [9] is to define a total Cauchy-like stress tensor, denoted here as $\boldsymbol{\tau}$, that incorporates in its definition the body forces, which are written as the divergence of a tensor field. Since from a practical point of view it is not possible to differentiate between loads of mechanical or magnetical origin inside an MS body, the above assumption would be valid. Let $\boldsymbol{T}$ denote the total nominal stress tensor (the transpose of the total Piola stress tensor), which is related to $\tau$ through [9]

$$
\boldsymbol{T}:=J \boldsymbol{F}^{-1} \boldsymbol{\tau} .
$$

Another key ingredient of the theory of Dorfmann and Ogden [9] is the assumption that there exists a total energy function $\Omega=\Omega\left(\boldsymbol{F}, \boldsymbol{B}_{l}, \boldsymbol{d}\right)$, where $\boldsymbol{d}$ is the preferred direction of a transversely isotropic material. ${ }^{1}$ It has been proven within the framework of magneto-elasticity that (see equations 3.11 and 3.13 of [9])

$$
\boldsymbol{T}=\frac{\partial \Omega}{\partial \boldsymbol{F}}, \quad \boldsymbol{H}_{l}=\frac{\partial \Omega}{\partial \boldsymbol{B}_{l}} .
$$


Using (6) and (3) $)_{1}$ we have

$$
\boldsymbol{\tau}=J^{-1} \boldsymbol{F} \frac{\partial \Omega}{\partial \boldsymbol{F}}, \quad \boldsymbol{H}=\boldsymbol{F}^{-\mathrm{T}} \frac{\partial \Omega}{\partial \boldsymbol{B}_{l}},
$$

and for incompressible MS bodies equation ( 8$)_{1}$ becomes

$$
\boldsymbol{\tau}=\boldsymbol{F} \frac{\partial \Omega}{\partial \boldsymbol{F}}-p \boldsymbol{I},
$$

where $p$ is the associated Lagrange multiplier due to the incompressibility constraint and $\boldsymbol{I}$ is the second-order identity tensor. The total stress tensor $\tau$ satisfies the localized force balance,

$$
\operatorname{div} \boldsymbol{\tau}=\mathbf{0},
$$

whereby mechanical body forces are neglected for brevity. Through the boundary surface $\partial \mathcal{B}_{t}$ and any discontinuity within $\mathcal{B}_{t}$ the magnetic variables and the total stress tensor must satisfy the following continuity conditions (see, for example, $[9,63])$ :

$$
\boldsymbol{n} \cdot \llbracket B \rrbracket=0, \quad \boldsymbol{n} \times \llbracket \boldsymbol{H} \rrbracket=\mathbf{0}, \quad \boldsymbol{\tau} \boldsymbol{n}=\hat{\boldsymbol{t}}+\boldsymbol{\tau}_{\mathrm{M}} \boldsymbol{n},
$$

where $\boldsymbol{n}$ is the unit outward normal vector to $\partial \mathcal{B}_{t}, \hat{\boldsymbol{t}}$ is the external mechanical traction, $\llbracket\{\bullet\} \rrbracket$ denotes the difference of a quantity from outside and inside a body, and $\boldsymbol{\tau}_{\mathrm{M}}$ is the Maxwell stress tensor outside the body defined as [63]

$$
\boldsymbol{\tau}_{\mathrm{M}}:=\boldsymbol{H} \otimes \boldsymbol{B}-\frac{1}{2}[\boldsymbol{H} \cdot \boldsymbol{B}] \boldsymbol{I} .
$$

\section{Spectral invariants for transversely isotropic magneto-elasticity}

In order to use the concept of spectral invariants, let us define the unit vector $\boldsymbol{a}$ in the direction of $\boldsymbol{B}_{l}$

$$
\boldsymbol{a}=\frac{1}{B} \boldsymbol{B}_{l} \quad \text { if } \quad B \neq 0 .
$$

where we have defined $B:=\left\|\boldsymbol{B}_{l}\right\|$. As outlined in the above, the magneto-elastic behavior can be characterized by the total energy function, which for reasons of objectivity is parameterized as

$$
\Omega=\hat{W}(\boldsymbol{C}, \boldsymbol{A}, \boldsymbol{D}, B)
$$

where $\boldsymbol{A}=\boldsymbol{a} \otimes \boldsymbol{a}, \boldsymbol{D}=\boldsymbol{d} \otimes \boldsymbol{d}$, and $\boldsymbol{d}$ is the direction of anisotropy of the MS elastomer in the reference configuration. Let $\lambda_{i}$ denote the eigenvalue of the right stretch tensor $\boldsymbol{U}$ and $\boldsymbol{e}_{i}$ its respective unitary eigendirection. The right Cauchy-Green tensor can be expressed in terms of the eigenvalues and unitary eigendirections as

$$
\boldsymbol{C}=\lambda_{1}^{2} \boldsymbol{E}_{1}+\lambda_{2}^{2} \boldsymbol{E}_{2}+\lambda_{3}^{2} \boldsymbol{E}_{3},
$$

with the eigen-dyads $\boldsymbol{E}_{i}=\boldsymbol{e}_{i} \otimes \boldsymbol{e}_{i}$. Thus, we can alternatively express the total energy function as

$$
\hat{W}(\boldsymbol{C}, \boldsymbol{A}, \boldsymbol{D}, B)=\bar{W}\left(\lambda_{1}, \lambda_{2}, \lambda_{3}, \boldsymbol{E}_{1}, \boldsymbol{E}_{2}, \boldsymbol{E}_{3}, \boldsymbol{A}, \boldsymbol{D}, B\right),
$$

with the symmetrical property

$$
\begin{gathered}
\bar{W}\left(\lambda_{1}, \lambda_{2}, \lambda_{3}, \boldsymbol{E}_{1}, \boldsymbol{E}_{2}, \boldsymbol{E}_{3}, \boldsymbol{A}, \boldsymbol{D}, B\right)=\bar{W}\left(\lambda_{2}, \lambda_{1}, \lambda_{3}, \boldsymbol{E}_{2}, \boldsymbol{E}_{1}, \boldsymbol{E}_{3}, \boldsymbol{A}, \boldsymbol{D}, B\right) \\
=\bar{W}\left(\lambda_{3}, \lambda_{2}, \lambda_{1}, \boldsymbol{E}_{3}, \boldsymbol{E}_{2}, \boldsymbol{E}_{1}, \boldsymbol{A}, \boldsymbol{D}, B\right)=\text { etc. }
\end{gathered}
$$

It is very important to know that $\Omega$ should be independent of $\boldsymbol{e}_{i}$ and $\boldsymbol{e}_{j}$ when $\lambda_{i}=\lambda_{j}, i \neq j$ in order for $\Omega$ to have a unique value, due to the non-unique values of $\boldsymbol{e}_{i}$ and $\boldsymbol{e}_{j}$ when $\lambda_{i}=\lambda_{j}$. Similarly, $\Omega$ should be independent 
of $\boldsymbol{e}_{1}, \boldsymbol{e}_{2}, \boldsymbol{e}_{3}$ when $\lambda_{1}=\lambda_{2}=\lambda_{3}$. We define this independent property as the $P$-property. We note that the total energy given in this paper satisfies the $P$-property.

Here $\bar{W}$ is an isotropic invariant of $\boldsymbol{E}_{1}, \boldsymbol{E}_{2}, \boldsymbol{E}_{3}, \boldsymbol{A}$, and $\boldsymbol{D}$, i.e.

$$
\begin{gathered}
\bar{W}\left(\lambda_{1}, \lambda_{2}, \lambda_{3}, \boldsymbol{E}_{1}, \boldsymbol{E}_{2}, \boldsymbol{E}_{3}, \boldsymbol{A}, \boldsymbol{D}, B\right) \\
=\bar{W}\left(\lambda_{1}, \lambda_{2}, \lambda_{3}, \boldsymbol{Q} \boldsymbol{E}_{1} \boldsymbol{Q}^{\mathrm{T}}, \boldsymbol{Q} \boldsymbol{E}_{2} \boldsymbol{Q}^{\mathrm{T}}, \boldsymbol{Q} \boldsymbol{E}_{3} \boldsymbol{Q}^{\mathrm{T}}, \boldsymbol{Q} \boldsymbol{A} \boldsymbol{Q}^{\mathrm{T}}, \boldsymbol{Q} \boldsymbol{D} \boldsymbol{Q}^{\mathrm{T}}, B\right)
\end{gathered}
$$

for all proper orthogonal tensors $\boldsymbol{Q} \in \mathrm{SO}(3)$. Taking note that $\operatorname{tr} \boldsymbol{E}_{i}=\operatorname{tr} \boldsymbol{A}=\operatorname{tr} \boldsymbol{D}=1, \boldsymbol{E}_{i}=\boldsymbol{E}_{i}^{2}=\boldsymbol{E}_{i}^{3}=\ldots$, $\boldsymbol{A}=\boldsymbol{A}^{2}=\boldsymbol{A}^{3}=\ldots, \boldsymbol{D}=\boldsymbol{D}^{2}=\boldsymbol{D}^{3}=\ldots$, and $\boldsymbol{E}_{i} \boldsymbol{E}_{j}=\mathbf{0}$ for $i \neq j$, and using the results of Spencer [54] for five second-order tensors, it follows that $\Omega$ can eventually be expressed as

$$
\Omega=\hat{\Omega}\left(\lambda_{1-3}, \zeta_{1-3}, \xi_{1-3}, \chi_{1-3}, \alpha, B\right)
$$

with the anisotropic spectral invariants $\zeta_{i}=\operatorname{tr}\left(\boldsymbol{E}_{i} \boldsymbol{A}\right)=\left[\boldsymbol{a} \bullet \boldsymbol{e}_{i}\right]^{2}, \xi_{i}=\operatorname{tr}\left(\boldsymbol{E}_{i} \boldsymbol{D}\right)=\left[\boldsymbol{d} \bullet \boldsymbol{e}_{i}\right]^{2}, \chi_{i}=\operatorname{tr}\left(\boldsymbol{E}_{i} \boldsymbol{A D}\right)=$ $\boldsymbol{a} \bullet \boldsymbol{d}\left[\boldsymbol{a} \bullet \boldsymbol{e}_{i}\right]\left[\boldsymbol{d} \bullet \boldsymbol{e}_{i}\right]$ and $\alpha=\operatorname{tr}(\boldsymbol{A D})=[\boldsymbol{a} \bullet \boldsymbol{d}]^{2}$.

In view of (17) we have the symmetrical property

$$
\begin{gathered}
\hat{\Omega}\left(\lambda_{1,2,3}, \zeta_{1,2,3}, \xi_{1,2,3}, \chi_{1,2,3}, \alpha, B\right)=\hat{\Omega}\left(\lambda_{2,1,3}, \zeta_{2,1,3}, \xi_{2,1,3}, \chi_{2,1,3}, \alpha, B\right) \\
\hat{\Omega}\left(\lambda_{3,2,1}, \zeta_{3,2,1}, \xi_{3,2,1}, \chi_{3,2,1}, \alpha, B\right)=\text { etc. }
\end{gathered}
$$

We note that the anisotropic spectral invariants $\zeta_{i}, \xi_{i}$, and $\chi_{i}$ (which depend on $\boldsymbol{e}_{i}$ ) do not have unique values if two or three eigenvalues of $\boldsymbol{U}$ coincide. However, relations among the different invariants are

$$
\zeta_{3}=1-\zeta_{1}-\zeta_{2}, \quad \xi_{3}=1-\xi_{1}-\xi_{2}, \text { and } \chi_{3}=\alpha-\chi_{2}-\chi_{3} .
$$

It is demonstrated by Shariff and Bustamante [59] that only seven of the thirteen invariants (ignore $B$ ) are independent. They also show that there are three syzygies and the minimal integrity basis

$$
S_{a}=\left\{\lambda_{1}, \lambda_{2}, \lambda_{3}, \zeta_{1}, \zeta_{2}, \xi_{1}, \xi_{2}, \chi_{1}, \chi_{2}, \alpha\right\}
$$

contains ten spectral invariants. This integrity basis shall be contrasted to the corresponding ten classical (principal) invariants for an MS transversely isotropic elastic body as

$$
\begin{gathered}
I_{1}=\operatorname{tr}(\boldsymbol{C}), \quad I_{2}=\frac{I_{1}^{2}-\operatorname{tr}\left(\boldsymbol{C}^{2}\right)}{2}, \quad I_{3}=\operatorname{det}(\boldsymbol{C}), I_{4}=\boldsymbol{a} \bullet \boldsymbol{C a}, I_{5}=\boldsymbol{a} \bullet \boldsymbol{C}^{2} \boldsymbol{a}, \\
I_{6}=\boldsymbol{d} \bullet \boldsymbol{C d}, \quad I_{7}=\boldsymbol{d} \bullet \boldsymbol{C}^{2} \boldsymbol{d}, I_{8}=[\boldsymbol{a} \bullet \boldsymbol{d}] \boldsymbol{a} \bullet \boldsymbol{C d}, I_{9}=[\boldsymbol{a} \bullet \boldsymbol{d}]^{2}, \\
I_{10}=[\boldsymbol{a} \bullet \boldsymbol{d}] \boldsymbol{a} \bullet \boldsymbol{C}^{2} \boldsymbol{d} .
\end{gathered}
$$

Next we express the classical (principal) invariants in terms of the novel spectral invariants, i.e. [59]

$$
\begin{gathered}
I_{1}=\lambda_{1}^{2}+\lambda_{2}^{2}+\lambda_{3}^{2}, \quad I_{2}=\lambda_{1}^{2} \lambda_{2}^{2}+\lambda_{1}^{2} \lambda_{3}^{2}+\lambda_{2}^{2} \lambda_{3}^{2}, \quad I_{3}=\left(\lambda_{1} \lambda_{2} \lambda_{3}\right)^{2}, \\
I_{4}=\lambda_{1}^{2} \zeta_{1}+\lambda_{2}^{2} \zeta_{2}+\lambda_{3}^{2} \zeta_{3}, \quad I_{5}=\lambda_{1}^{4} \zeta_{1}+\lambda_{2}^{4} \zeta_{2}+\lambda_{3}^{4} \zeta_{3}, \\
I_{6}=\lambda_{1}^{2} \xi_{1}+\lambda_{2}^{2} \xi_{2}+\lambda_{3}^{2} \xi_{3}, \quad I_{7}=\lambda_{1}^{4} \xi_{1}+\lambda_{2}^{4} \xi_{2}+\lambda_{3}^{4} \xi_{3}, \\
I_{8}=\sum_{i=1}^{3} \lambda_{i}^{2} \chi_{i}, \quad I_{9}=[\boldsymbol{a} \bullet \boldsymbol{d}]^{2}, \quad I_{10}=\sum_{i=1}^{3} \lambda_{i}^{4} \chi_{i} .
\end{gathered}
$$


For the constitutive behavior of compressible and incompressible MS bodies in terms of $\boldsymbol{C}$ we have, respectively,

$$
\boldsymbol{\tau}=2 J^{-1} \boldsymbol{F} \frac{\partial \Omega}{\partial \boldsymbol{C}} \boldsymbol{F}^{\mathrm{T}}, \quad \boldsymbol{\tau}=2 \boldsymbol{F} \frac{\partial \Omega}{\partial \boldsymbol{C}} \boldsymbol{F}^{\mathrm{T}}-p \boldsymbol{I} .
$$

The Lagrangian spectral components of the Piola-Kirchhoff stress resulting from this relation are computed following the work of Shariff [57]. The normal components result in

$$
\left(\frac{\partial \Omega}{\partial C}\right)_{i i}=\frac{1}{2 \lambda_{i}} \frac{\partial \hat{\Omega}}{\partial \lambda_{i}} \quad(\text { no sum on } i)
$$

whereas the shear components are expressed as

$$
\begin{aligned}
\left(\frac{\partial \Omega}{\partial \boldsymbol{C}}\right)_{i j} & =\frac{1}{\left[\lambda_{i}^{2}-\lambda_{j}^{2}\right]}\left\{\left[\frac{\partial \hat{\Omega}}{\partial \zeta_{i}}-\frac{\partial \hat{\Omega}}{\partial \zeta_{j}}\right] \boldsymbol{e}_{i} \bullet \boldsymbol{A} \boldsymbol{e}_{j}+\left[\frac{\partial \hat{\Omega}}{\partial \xi_{i}}-\frac{\partial \hat{\Omega}}{\partial \xi_{j}}\right] \boldsymbol{e}_{i} \bullet \boldsymbol{D} \boldsymbol{e}_{j}\right. \\
& \left.+\left[\frac{\partial \hat{\Omega}}{\partial \chi_{i}}-\frac{\partial \hat{\Omega}}{\partial \chi_{j}}\right]\left[\boldsymbol{e}_{i} \bullet \boldsymbol{A D} \boldsymbol{e}_{j}+\boldsymbol{e}_{j} \bullet \boldsymbol{A D} \boldsymbol{e}_{i}\right]\right\}, \quad i \neq j .
\end{aligned}
$$

Consequently the Eulerian spectral components of $\tau$ are

$$
\begin{aligned}
\tau_{i i} & =\frac{2 \lambda_{i}^{2}}{J}\left(\frac{\partial \Omega}{\partial \boldsymbol{C}}\right)_{i i},(\text { no sum on } i) \\
\tau_{i j} & =\frac{2 \lambda_{i} \lambda_{j}}{J}\left(\frac{\partial \Omega}{\partial \boldsymbol{C}}\right)_{i j}, \quad i \neq j
\end{aligned}
$$

for compressible materials, while for incompressible MS bodies since $J=1$ from $(25)_{2}$, we have that

$$
\begin{aligned}
\tau_{i i} & =2 \lambda_{i}^{2}\left(\frac{\partial \Omega}{\partial \boldsymbol{C}}\right)_{i i}-p,(\text { no sum on } i) \\
\tau_{i j} & =2 \lambda_{i} \lambda_{j}\left(\frac{\partial \Omega}{\partial \boldsymbol{C}}\right)_{i j}, \quad i \neq j .
\end{aligned}
$$

The corresponding relation for the Lagrangian magnetic field is given by

$$
\boldsymbol{H}_{l}=\frac{\partial \Omega}{\partial \boldsymbol{B}_{l}}=\sum_{k=1}^{3} H_{k} \boldsymbol{e}_{k},
$$

where

$$
\begin{gathered}
H_{k}=\left[\boldsymbol{a} \bullet \boldsymbol{e}_{k}\right]\left[\frac{\partial \hat{\Omega}}{\partial B}+\frac{2}{B}\left[\frac{\partial \hat{\Omega}}{\partial \zeta_{k}}-\sum_{i=1}^{3} \frac{\partial \hat{\Omega}}{\partial \zeta_{i}} \zeta_{i}-\sum_{i=1}^{3} \frac{\partial \hat{\Omega}}{\partial \chi_{i}} \chi_{i}-\alpha \frac{\partial \hat{\Omega}}{\partial \alpha}\right]\right] \\
+\frac{\left[\boldsymbol{d} \bullet \boldsymbol{e}_{k}\right]}{B}\left[\sum_{i=1}^{3}\left[\boldsymbol{a} \bullet \boldsymbol{e}_{i}\right]\left[\boldsymbol{d} \bullet \boldsymbol{e}_{i}\right] \frac{\partial \hat{\Omega}}{\partial \chi_{i}}+[\boldsymbol{a} \bullet \boldsymbol{d}] \frac{\partial \hat{\Omega}}{\partial \chi_{k}}+2[\boldsymbol{a} \bullet \boldsymbol{d}] \frac{\partial \hat{\Omega}}{\partial \alpha}\right] .
\end{gathered}
$$

Consequently, the Eulerian magnetic field in the deformed configuration can be expressed by

$$
\boldsymbol{H}=\boldsymbol{F}^{-T} \frac{\partial \Omega}{\partial \boldsymbol{B}_{l}}=\sum_{k=1}^{3} \frac{H_{k}}{\lambda_{k}} \boldsymbol{v}_{k},
$$

where $\boldsymbol{v}_{k}$ is the unitary eigendirection of the left stretch tensor $\boldsymbol{V}$, i.e. $\boldsymbol{V}_{k}:=\lambda_{k}^{-1} \boldsymbol{F}^{-T} \boldsymbol{e}_{k}$. 


\section{I. The case of a rigid magnetic permeator}

An interesting special case to consider is a rigid magnetic permeator where an external magnetic induction is applied to the body, but there is no deformation, i.e. $\boldsymbol{F}=\boldsymbol{I}$, which implies $\lambda_{1}=\lambda_{2}=\lambda_{3}=1$. Here we choose, without loss of generality, $\boldsymbol{a}=\boldsymbol{e}_{3}$ and $\boldsymbol{d}$ perpendicular to $\boldsymbol{e}_{2}$. Hence, $\zeta_{3}=1, \zeta_{1}=\zeta_{2}=0, \xi_{2}=0, \xi_{1}=1-\xi_{3}$, $\chi_{1}=\chi_{2}=0, \chi_{3}=\xi_{3}$, and $\alpha=\xi_{3}$. Thus, the total Cauchy stress induced by the external magnetic induction is

$$
\begin{aligned}
\boldsymbol{\tau}_{0} & =\sum_{i=1}^{3} \frac{\partial \hat{\Omega}}{\partial \lambda_{i}}\left(1,1,1,0,0,1,1-\xi_{3}, 0, \xi_{3}, 0,0, \xi_{3}, \xi_{3}, B\right) \boldsymbol{e}_{i} \otimes \boldsymbol{e}_{i} \\
& +L\left[\boldsymbol{e}_{1} \bullet \boldsymbol{d}\right]\left[\boldsymbol{e}_{3} \bullet \boldsymbol{d}\right]\left[\boldsymbol{e}_{1} \otimes \boldsymbol{e}_{3}+\boldsymbol{e}_{3} \otimes \boldsymbol{e}_{1}\right]-p \boldsymbol{I},
\end{aligned}
$$

where

$$
L=\lim _{\lambda_{1}, \lambda_{3} \rightarrow 1} \frac{1}{\left[\lambda_{1}^{2}-\lambda_{3}^{2}\right]}\left[\frac{\partial \hat{\Omega}}{\partial \xi_{1}}-\frac{\partial \hat{\Omega}}{\partial \xi_{3}}+\frac{\partial \hat{\Omega}}{\partial \chi_{1}}-\frac{\partial \hat{\Omega}}{\partial \chi_{3}}\right]
$$

assuming the limit exists. The corresponding magnetic field in the undeformed configuration can be expressed as

$$
\boldsymbol{H}_{0}=\frac{\partial \hat{\Omega}}{\partial B} \boldsymbol{a}+\frac{1}{B} \frac{\partial \hat{\Omega}}{\partial \chi_{1}}[\boldsymbol{a} \bullet \boldsymbol{d}]\left[\boldsymbol{e}_{1} \bullet \boldsymbol{d}\right] \boldsymbol{e}_{1}+\left[\frac{\partial \hat{\Omega}}{\partial \chi_{3}}+\frac{2}{B} \frac{\partial \hat{\Omega}}{\partial \alpha}\right][[\boldsymbol{a} \bullet \boldsymbol{d}] \boldsymbol{d}-\alpha \boldsymbol{a}] .
$$

For a deformable permeator $\boldsymbol{\tau}_{0}$ denotes the total Cauchy stress required to prevent deformation due to the magnetic load.

\section{Solution of some boundary value problems}

In this section, we analytically solve some boundary value problems considering the generic expressions for the magneto-elastic constitutive equations using the novel spectral invariants. We only consider incompressible solids for which the energy function can be written as

$$
\tilde{\Omega}\left(\lambda_{1}, \lambda_{2}, \zeta_{1-3}, \xi_{1-3}, \chi_{1-3}, \alpha, B\right)=\hat{\Omega}\left(\lambda_{1}, \lambda_{2}, \lambda_{3}=\lambda_{1}^{-1} \lambda_{2}^{-1}, \zeta_{1-3}, \xi_{1-3}, \chi_{1-3}, \alpha, B\right) .
$$

Here the following symmetry will be taken into account:

$$
\tilde{\Omega}\left(\lambda_{1}, \lambda_{2}, \zeta_{1}, \zeta_{2}, \zeta_{3}, \xi_{1}, \xi_{2}, \xi_{3}, \chi_{1}, \chi_{2}, \chi_{3}, \alpha, B\right)=\tilde{\Omega}\left(\lambda_{2}, \lambda_{1}, \zeta_{2}, \zeta_{1}, \zeta_{3}, \xi_{2}, \xi_{1}, \xi_{3}, \chi_{2}, \chi_{1}, \chi_{3}, \alpha, B\right)
$$

\section{I. Triaxial extension of a slab}

4.I.I. General setting. We consider a pure homogeneous deformation defined by

$$
x_{1}=\lambda_{1} X_{1}, \quad x_{2}=\lambda_{2} X_{2}, \quad x_{3}=\lambda_{3} X_{3},
$$

where $x_{i}$ and $X_{i}$ are the Cartesian components of $\boldsymbol{x}$ and $\boldsymbol{X}$, respectively. For this deformation $\mathrm{F}=\mathrm{U} \equiv$ $\operatorname{diag}\left(\lambda_{1}, \lambda_{2}, \lambda_{3}\right)$ and the Lagrangian and Eulerian axes of the deformation coincide with the Cartesian coordinate directions and are fixed as the values of the stretches change. Here we consider a slab of material of uniform finite thickness $h$, with (plane) faces normal to the $X_{3}$ direction and very large in the $X_{1}$ and $X_{2}$ directions, i.e. the slab is defined by $-h / 2 \leq X_{3} \leq h / 2,-L \leq X_{1} \leq L,-L \leq X_{2} \leq L$, where $h \ll L$, cf. Figure 1. We assume the slab is subjected to a magnetic induction $\boldsymbol{B}_{l}$, where, for simplicity, we consider both $B$ and $\boldsymbol{a}$ independent of $\boldsymbol{X}$ so that (2) is satisfied automatically.

From (30) and (31) it is possible to show that the total Cauchy stress components in Cartesian coordinates take the form

$$
\tau_{11}-\tau_{33}=\lambda_{1} \frac{\partial \tilde{\Omega}}{\partial \lambda_{1}}, \quad \tau_{22}-\tau_{33}=\lambda_{2} \frac{\partial \tilde{\Omega}}{\partial \lambda_{2}}
$$




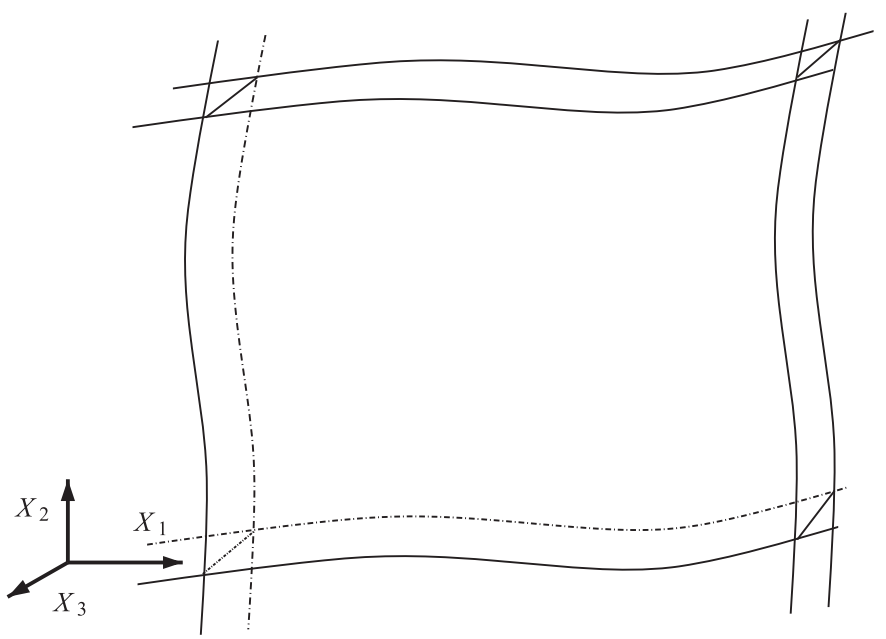

Figure I. A very thin slab where the two very large dimensions, i.e. $X_{1}$ and $X_{2}$, are truncated.

$$
\tau_{i j}=2 \lambda_{i} \lambda_{j}\left(\frac{\partial \Omega}{\partial \boldsymbol{C}}\right)_{i j}, \quad i \neq j
$$

The total Cauchy stress is constant and so (10) is also satisfied automatically.

4.I.2. Special case. When the direction of the magnetic induction is such that $\boldsymbol{a}=\boldsymbol{d}=\boldsymbol{e}_{3}$ (i.e. the field is applied in the direction of the thickness of the plate), we have $\zeta_{3}=\xi_{3}=\chi_{3}=\alpha=1, \zeta_{1}=\zeta_{2}=\xi_{1}=\xi_{2}=\chi_{1}=\chi_{2}=$ 0 . Inserting these values in (40) and (41), we obtain

$$
\tau_{11}-\tau_{33}=\lambda_{1} \frac{\partial \tilde{\Omega}}{\partial \lambda_{1}}, \quad \tau_{22}-\tau_{33}=\lambda_{2} \frac{\partial \tilde{\Omega}}{\partial \lambda_{2}}, \quad \tau_{12}=\tau_{23}=\tau_{13}=0,
$$

while from (13), (3) 1 , and (5) we have

$$
\boldsymbol{B}=\lambda_{3} B \boldsymbol{e}_{3}, \quad \boldsymbol{H}=\frac{1}{\lambda_{3}} \frac{\partial \tilde{\Omega}}{\partial B} \boldsymbol{e}_{3}, \quad \boldsymbol{M}=\left[\lambda_{3} B-\frac{\mu_{0}}{\lambda_{3}} \frac{\partial \tilde{\Omega}}{\partial B}\right] \boldsymbol{e}_{3} .
$$

When we assume the slab is surrounded by a vacuum, considering the continuity conditions $(11)_{1,2}$ for the surfaces $X_{3}= \pm h / 2$ the magnetic induction in a vacuum must be the same as within the slab (see equation $\left.(11)_{1}\right)$. Therefore, from (4) and (12) the non-zero Cartesian components of the Maxwell stress tensor are

$$
\tau_{M_{11}}=\tau_{M_{22}}=-\frac{1}{2 \mu_{0}} B^{2} \lambda_{3}^{2}=-\tau_{M_{33}}
$$

If no mechanical traction is supplied to the plane faces of the slab $X_{3}= \pm h / 2$, then from $(11)_{3}$ we have $\tau_{33}=\tau_{M_{33}}$ and from (42) we have

$$
\tau_{11}=\frac{1}{2 \mu_{0}} B^{2} \lambda_{3}^{2}+\lambda_{1} \frac{\partial \tilde{\Omega}}{\partial \lambda_{1}}, \quad \tau_{22}=\frac{1}{2 \mu_{0}} B^{2} \lambda_{3}^{2}+\lambda_{2} \frac{\partial \tilde{\Omega}}{\partial \lambda_{2}} .
$$

Since $L \gg h$ as an approximation in the above case, we do not require (11) to be satisfied across the surfaces $X_{1}= \pm L$ and $X_{2}= \pm L$, i.e. for a very large plate in the directions 1 and 2 we are neglecting "edge" effects, and assuming then that the uniform field (39), (43) is a solution of the boundary value problem.

Let us specialize to the case of equibiaxial deformation, where $\lambda_{1}=\lambda_{2}=\lambda$ and $\lambda_{3}=\lambda^{-2}$, from (45) we obtain

$$
\tau_{11}=\tau_{22}=\frac{1}{2 \mu_{0} \lambda^{4}} B^{2}+\lambda \frac{\partial \tilde{\Omega}}{\partial \lambda_{1}}(\lambda, \lambda, 0,0,1,0,0,1,0,0,1,1, B)
$$




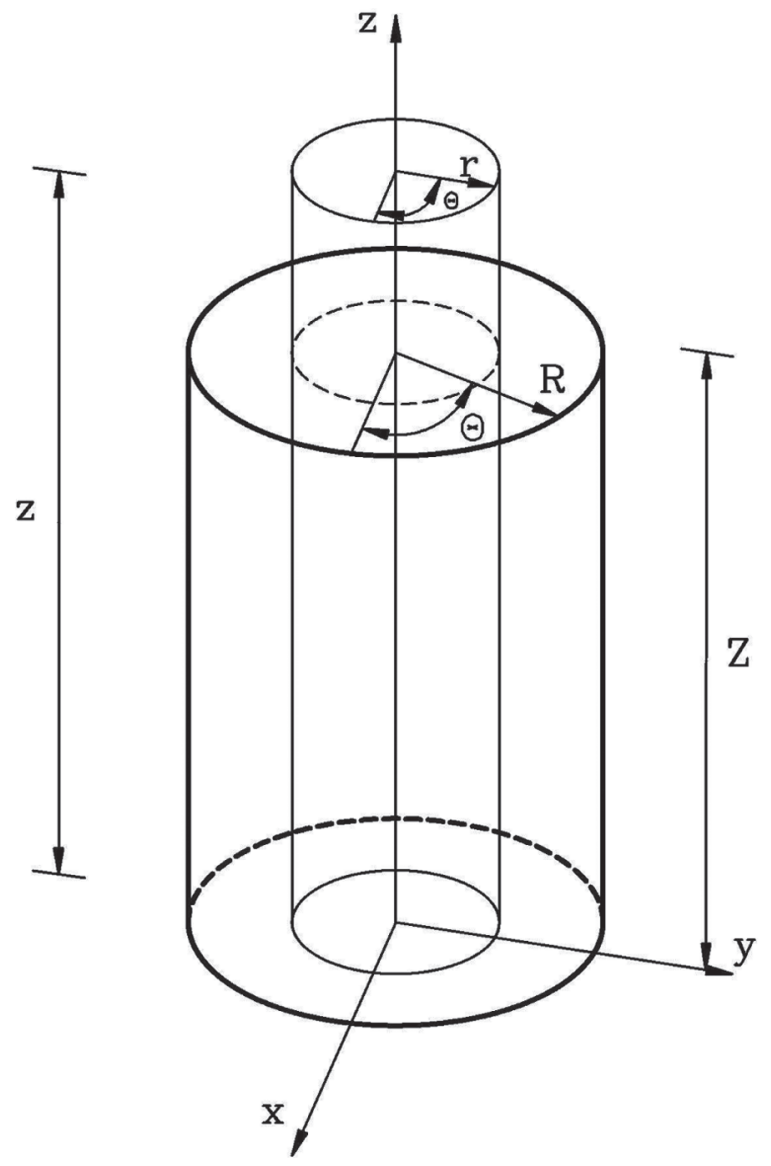

Figure 2. A cylindrical body: material and spatial configurations.

taking note that due to the symmetry in $\tilde{\Omega}$

$$
\frac{\partial \tilde{\Omega}}{\partial \lambda_{1}}(\lambda, \lambda, 0,0,1,0,0,1,0,0,1,1, B)=\frac{\partial \tilde{\Omega}}{\partial \lambda_{2}}(\lambda, \lambda, 0,0,1,0,0,1,0,0,1,1, B) .
$$

Finally, for a rigid magnetic permeator, we have

$$
\tau_{0_{11}}=\tau_{0_{22}}=\frac{1}{2 \mu_{0}} B^{2}+\frac{\partial \tilde{\Omega}}{\partial \lambda_{1}}(1,1,0,0,1,0,0,1,0,0,1,1, B) .
$$

Recall that this is the initial lateral stress needed to prevent deformation due to the magnetic field.

\subsection{Extension and simple torsion of a circular cylinder}

4.2.I. General setting. The problem of extension and torsion of a cylinder has been studied previously by Bustamante [2] using the standard principal invariants. The tube's initial geometry is defined by

$$
0 \leq R \leq R_{0}, \quad 0 \leq \Theta \leq 2 \pi, \quad-\infty<Z<\infty,
$$

where $R, \Theta$, and $Z$ are cylindrical polar coordinates in the undeformed configuration, cf. Figure 2 . The deformation is described by

$$
r=\lambda^{-\frac{1}{2}} R, \quad \theta=\Theta+\lambda \tau Z, \quad z=\lambda Z,
$$


where $\tau$ is the amount of torsional twist per unit deformed length and $\lambda$ is the axial stretch. In the above formulation, $r, \theta$, and $z$ are cylindrical polar coordinates in the deformed configuration. The components of the deformation gradient in Cartesian coordinates are

$$
\boldsymbol{F} \equiv\left[\begin{array}{ccc}
\lambda^{-\frac{1}{2}} & 0 & 0 \\
0 & \lambda^{-\frac{1}{2}} & \lambda \gamma \\
0 & 0 & \lambda
\end{array}\right]
$$

where $\gamma=r \tau$ and, in this paper, we only consider $\lambda \geq 1$. The Lagrangian axes (eigendirections) have cylindrical polar components:

$$
\boldsymbol{e}_{1} \equiv\left[\begin{array}{l}
1 \\
0 \\
0
\end{array}\right], \quad \boldsymbol{e}_{2} \equiv\left[\begin{array}{l}
0 \\
c \\
s
\end{array}\right], \quad \boldsymbol{e}_{3} \equiv\left[\begin{array}{c}
0 \\
-s \\
c
\end{array}\right]
$$

where

$$
\begin{gathered}
c=\cos (\phi)=\frac{2}{\sqrt{2\left[\hat{\gamma}^{2}+4\right]+2 \hat{\gamma} \sqrt{\hat{\gamma}^{2}+4}}}, \quad s=\sin (\phi)=\frac{\hat{\gamma}+\sqrt{\hat{\gamma}^{2}+4}}{\sqrt{2\left[\hat{\gamma}^{2}+4\right]+2 \hat{\gamma} \sqrt{\hat{\gamma}^{2}+4}}}, \\
\frac{\pi}{4} \leq \frac{\pi-\tan ^{-1}\left(\frac{1}{\sqrt{\lambda^{3}-1}}\right)}{2} \leq \phi<\frac{\pi}{2},
\end{gathered}
$$

and

$$
\hat{\gamma}=\frac{\lambda^{3} \gamma^{2}+\lambda^{3}-1}{\lambda^{\frac{3}{2}} \gamma} \geq 0 .
$$

We also have the relation

$$
c^{2}-s^{2}=-\hat{\gamma} c s
$$

In the case of simple torsion $\lambda=1$, and thus $\hat{\gamma}=\gamma$. The principal stretches for a combined extension and torsion deformation have the forms

$$
\begin{gathered}
\lambda_{1}=\frac{1}{\lambda^{\frac{1}{2}}}, \quad \lambda_{2}=\sqrt{\frac{\lambda^{3}\left[1+\gamma^{2}\right]+1+\lambda^{\frac{3}{2}} \gamma \sqrt{\hat{\gamma}^{2}+4}}{2 \lambda}}=\sqrt{\frac{1}{\lambda}+\frac{s \gamma \sqrt{\lambda}}{c},} \\
\lambda_{3}=\sqrt{\frac{\lambda^{3}\left[1+\gamma^{2}\right]+1-\lambda^{\frac{3}{2}} \gamma \sqrt{\hat{\gamma}^{2}+4}}{2 \lambda}}=\sqrt{\frac{1}{\lambda}-\frac{c \gamma \sqrt{\lambda}}{s} .}
\end{gathered}
$$

Finally, the cylindrical polar components of the total Cauchy stress take the form

$$
\begin{gathered}
\tau_{\theta \theta}=-p+2\left[\frac{l_{2} c^{2}+l_{3} s^{2}-2 l_{6} c s}{\lambda}+2 \sqrt{\lambda} \gamma\left[\left[l_{2}-l_{3}\right] c s+l_{6}\left[c^{2}-s^{2}\right]\right]+\lambda^{2} \gamma^{2}\left[l_{2} s^{2}+l_{3} c^{2}+2 l_{6} c s\right]\right] \\
\tau_{z \theta}=2\left[\sqrt{\lambda}\left[\left[l_{2}-l_{3}\right] c s+l_{6}\left[c^{2}-s^{2}\right]\right]+\lambda^{2} \gamma\left[l_{2} s^{2}+l_{3} c^{2}+2 l_{6} c s\right]\right] \\
\tau_{z z}=-p+2 \lambda^{2}\left[l_{2} s^{2}+l_{3} c^{2}+2 l_{6} c s\right]
\end{gathered}
$$




$$
\begin{gathered}
\tau_{r \theta}=2\left[\frac{l_{4} c-l_{5} s}{\lambda}+\sqrt{\lambda} \gamma\left[l_{4} s+l_{5} c\right]\right], \\
\tau_{r r}=-p+\frac{2 l_{1}}{\lambda}, \\
\tau_{z r}=2 \sqrt{\lambda}\left[l_{4} s+l_{5} c\right],
\end{gathered}
$$

where

$$
\begin{gathered}
l_{1}=\frac{1}{2 \lambda_{1}} \frac{\partial \tilde{\Omega}}{\partial \lambda_{1}}, \quad l_{2}=\frac{1}{2 \lambda_{2}} \frac{\partial \tilde{\Omega}}{\partial \lambda_{2}}, \quad l_{3}=\frac{1}{2} \frac{\partial \tilde{\Omega}}{\partial \lambda_{3}}, \\
l_{4}=\left(\frac{\partial \tilde{\Omega}}{\partial \boldsymbol{C}}\right)_{12}, \quad l_{5}=\left(\frac{\partial \tilde{\Omega}}{\partial \boldsymbol{C}}\right)_{13}, \quad l_{6}=\left(\frac{\partial \tilde{\Omega}}{\partial \boldsymbol{C}}\right)_{23} .
\end{gathered}
$$

Note that the expressions in (59) can be obtained from the general equations in (27) and (37).

4.2.2. Special case. Next consider the special case when $\boldsymbol{a}=\boldsymbol{d} \equiv[0,0,1]^{\mathrm{T}}$. In this condition $l_{4}=l_{5}=0$ and hence $\tau_{r z}=\tau_{r \theta}=0$. The spectral invariants have the values $\alpha=1, \zeta_{1}=\xi_{1}=\chi_{1}=0, \zeta_{2}=\xi_{2}=\chi_{2}=s^{2}$, and $\zeta_{3}=\xi_{3}=\chi_{3}=c^{2}$. The magnetic field relations then have the form

$$
\begin{aligned}
\boldsymbol{H}_{1}= & \frac{\partial \hat{\Omega}}{\partial B} \boldsymbol{a}+\frac{2}{B}\left[\frac{\partial \hat{\Omega}}{\partial \zeta_{2}}\left[s \boldsymbol{e}_{2}-s^{2} \boldsymbol{a}\right]+\frac{\partial \hat{\Omega}}{\partial \zeta_{3}}\left[c \boldsymbol{e}_{3}-c^{2} \boldsymbol{a}\right]\right] \\
& +\frac{1}{B}\left[\frac{\partial \hat{\Omega}}{\partial \chi_{2}}\left[s \boldsymbol{e}_{2}-s^{2} \boldsymbol{a}\right]+\frac{\partial \hat{\Omega}}{\partial \chi_{3}}\left[c \boldsymbol{e}_{3}-c^{2} \boldsymbol{a}\right]\right] .
\end{aligned}
$$

The Maxwell stress in cylindrical polar components composes as

$$
\boldsymbol{\tau}_{M} \equiv \mu_{0}^{-1} B^{2}\left[\begin{array}{ccc}
-\frac{1+\gamma^{2}}{2} & 0 & 0 \\
0 & \frac{\gamma^{2}-1}{2} & \gamma \\
0 & \gamma & \frac{1-\gamma^{2}}{2}
\end{array}\right] .
$$

Since the deformation depends on $\gamma$ and $\lambda$, the energy function can be considered as a function of $\gamma, \lambda$ and $B$, i.e. $\hat{\Omega}=\bar{\Omega}(\lambda, \gamma, B)$. We can easily deduce that,

$$
\tau_{z \theta}=\frac{\partial \bar{\Omega}}{\partial \gamma} .
$$

In order to obtain the above relation (62) we require the following formulae

$$
\frac{\partial \lambda_{2}}{\partial \gamma}=\frac{1}{\lambda_{2}}\left[s c \sqrt{\lambda}+\lambda^{2} \gamma s^{2}\right], \quad \frac{\partial \lambda_{3}}{\partial \gamma}=\frac{1}{\lambda_{3}}\left[-s c \sqrt{\lambda}+\lambda^{2} \gamma c^{2}\right],
$$

and

$$
\frac{\partial s^{2}}{\partial \gamma}=-\frac{\partial c^{2}}{\partial \gamma}=\frac{s c}{\lambda_{2}^{2}-\lambda_{3}^{2}}\left[2 \sqrt{\lambda}\left[c^{2}-s^{2}\right]+4 \lambda^{2} \gamma s c\right] .
$$




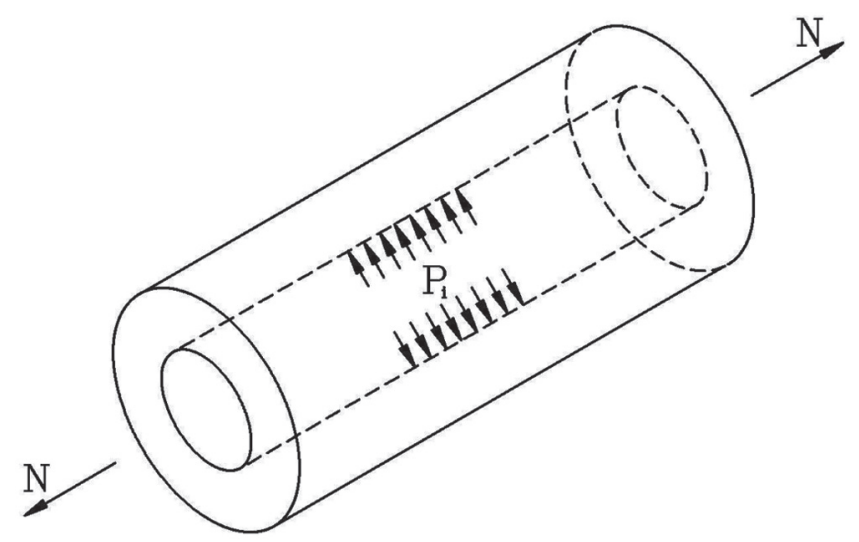

Figure 3. A cylindrical tube being inflated under an internal pressure $P_{i}$ while it is extended by an axially applied force $N$.

Using the equilibrium equation

$$
\tau_{r r}+\tau_{\theta \theta}=\frac{1}{r} \frac{d\left(r^{2} \tau_{33}\right)}{d r}
$$

and the assumption that no mechanical stress is applied at the surface $r=a$, the mechanical axial force $N$ and the torque $M$, i.e. the stress resultants, applied at the ends of the cylinder are as follows:

$$
\begin{gathered}
N=\pi \int_{0}^{a}\left[2 \tau_{z z}-\tau_{r r}-\tau_{\theta \theta}\right] r d r-\pi a^{2} \mu_{0}^{-1} B^{2} \frac{1-\gamma^{2}}{2}, \\
M=2 \pi \int_{0}^{a} \frac{\partial \bar{\Omega}}{\partial \gamma} r^{2} d r .
\end{gathered}
$$

\subsection{Extension and inflation of a thick-walled tube}

4.3.I. General setting. In this last example we study the problem of an incompressible MS thick-walled circular cylindrical tube under inflation and extension, see Figure 3. The tube's initial geometry is defined by

$$
R_{\mathrm{i}} \leq R \leq R_{\mathrm{o}}, \quad 0 \leq \Theta \leq 2 \pi, \quad 0 \leq Z \leq L,
$$

where $R_{\mathrm{i}}, R_{\mathrm{o}}, L$ are positive constants and $R, \Theta, Z$ are cylindrical polar coordinates. We assume the tube is deformed as

$$
r^{2}-r_{\mathrm{i}}^{2}=\frac{1}{\lambda_{z}}\left[R^{2}-R_{\mathrm{i}}^{2}\right], \quad \theta=\Theta, \quad z=\lambda_{z} Z,
$$

where $r_{\mathrm{i}}$ is the internal radius of the deformed tube, $r, \theta$, and $z$ are cylindrical polar coordinates in the deformed configuration and $\lambda_{z}>0$ (constant) is the axial stretch. The principal stretches for this deformation are given by

$$
\lambda_{1}=\frac{1}{\lambda \lambda_{z}}, \quad \lambda_{2}=\lambda=\frac{r}{R}, \quad \lambda_{3}=\lambda_{z},
$$

It can be easily shown that $F \equiv \operatorname{diag}\left(1 /\left[\lambda \lambda_{z}\right], \lambda, \lambda_{z}\right)$ and the Lagrangian axes (eigendirections) are

$$
\boldsymbol{e}_{1}=\boldsymbol{E}_{R}, \quad \boldsymbol{e}_{2}=\boldsymbol{E}_{\Theta}, \quad \boldsymbol{e}_{3}=\boldsymbol{E}_{Z},
$$

where $\boldsymbol{E}_{R}, \boldsymbol{E}_{\Theta}, \boldsymbol{E}_{Z}$ are the base vectors for the $R, \Theta, Z$ cylindrical coordinate system. Consider the function

$$
\Omega_{E}\left(\lambda, \lambda_{z}, \zeta_{1-3}, \xi_{1-3}, \chi_{1-3}, \alpha, B\right)=\hat{\Omega}\left(\frac{1}{\lambda \lambda_{z}}, \lambda, \lambda_{z}, \zeta_{1-3}, \xi_{1-3}, \chi_{1-3}, \alpha, B\right) .
$$


Finally from (30) and (31), we have

$$
\tau_{\theta \theta}-\tau_{r r}=\lambda \frac{\partial \Omega_{E}}{\partial \lambda}, \quad \tau_{z z}-\tau_{r r}=\lambda_{z} \frac{\partial \Omega_{E}}{\partial \lambda_{z}} .
$$

4.3.2. Special cases. Consider the case when $\boldsymbol{d} \equiv[0,0,1]^{\mathrm{T}}$ and the magnetic induction $\boldsymbol{B}_{l}=B \boldsymbol{a}$ is such that

$$
\boldsymbol{a}=\cos (\alpha) \boldsymbol{E}_{\Theta}+\sin (\alpha) \boldsymbol{E}_{Z}=\cos (\alpha) \boldsymbol{e}_{2}+\sin (\alpha) \boldsymbol{e}_{3},
$$

where $0 \leq \alpha \leq \frac{\pi}{2}$ and $\zeta_{1}=0$ and let us assume that in general $B=B(r)$. Let us discuss how to obtain such a field in reality. The component in the axial direction $Z$ could be generated by permanent magnets located at $Z=0$ and $Z=L$, while the component in the direction $\Theta$ could be generated using a wire carrying an electric current going in a spiral coil around the tube in the axial direction. The total Cauchy shear stress components take the values

$$
\tau_{\theta z}=\frac{2 \lambda \lambda_{z} c s}{\lambda^{2}-\lambda_{z}^{2}}\left[\frac{\partial \Omega_{E}}{\partial \zeta_{2}}-\frac{\partial \Omega_{E}}{\partial \zeta_{3}}+\frac{\partial \Omega_{E}}{\partial \chi_{2}}-\frac{\partial \Omega_{E}}{\partial \chi_{3}}\right], \quad \tau_{r z}=\tau_{r \theta}=0
$$

It is clear that when $\alpha=0$ or $\alpha=\frac{\pi}{2}$ the shear stress $\tau_{\theta z}$ is zero.

By considering the symmetry of the problem, the equilibrium equation (10) with negligible body forces reduces to

$$
\frac{d \tau_{r r}}{d r}+\frac{1}{r}\left[\tau_{r r}-\tau_{\theta \theta}\right]=0
$$

and from (73) and (76) we have the solution

$$
\tau_{r r}=\int_{r_{\mathrm{i}}}^{r} \lambda(\xi) \frac{\partial \tilde{\Omega}}{\partial \lambda}(\xi) \mathrm{d} \xi+\tau_{r r_{i}},
$$

where $\lambda(\xi)=\xi / R(\xi)$ and $R(\xi)$ is obtained from (69) $)_{1}$ replacing $r$ by the auxiliary variable $\xi$, and $\tau_{r r_{i}}$ is the value of $\tau_{r r}$ at $r=r_{\mathrm{i}}$.

Let us discuss now the magnetic variables and the continuity conditions (11). Using the expression for $\boldsymbol{F}$ and $\boldsymbol{B}_{l}$ from (69) and (74). In view of (33) and (34) we obtain

$$
\boldsymbol{B} \equiv B\left[\begin{array}{c}
0 \\
\lambda \cos \alpha \\
\lambda_{z} \sin \alpha
\end{array}\right], \quad \boldsymbol{H} \equiv\left[\begin{array}{c}
0 \\
H_{\theta}(r) \\
H_{z}(r)
\end{array}\right]
$$

where, unlike the problems presented in Sections 4.1 and 4.2, $B$ is not constant and it can depend on $r$. For brevity we do not present the full expressions for the components of $\boldsymbol{H}$, but for this problem it is easy to see from (34) that they depend on $r$ and $B=B(r)$. For this expression of $\boldsymbol{B}$, equation (2) (in cylindrical coordinates) is satisfied automatically, but $(2)_{2}$ becomes

$$
\frac{1}{r} \frac{\mathrm{d}}{\mathrm{d} r}\left(r H_{\theta}\right)=0 \quad \text { and } \quad \frac{\mathrm{d} H_{z}}{\mathrm{~d} r}=0 .
$$

In general both equations cannot be satisfied at the same time for the same $B=B(r)$. As an illustration, let us assume two cases: $\alpha=0$ and $\alpha=\pi / 2$ in (74).

Case $\alpha=0$ : In this case $\boldsymbol{B}=B \lambda \boldsymbol{e}_{\theta}$ and $\boldsymbol{a}=\boldsymbol{E}_{\theta}$, then considering that $\boldsymbol{d}=\boldsymbol{E}_{z}$ from (33) we have $H_{z}=0$ and

$$
H_{\theta}=\frac{\partial \hat{\Omega}}{\partial B}+\frac{2}{B}\left[\frac{\partial \hat{\Omega}}{\partial \zeta_{2}}-\sum_{i=1}^{3} \frac{\partial \hat{\Omega}}{\partial \zeta_{i}} \zeta_{i}-\sum_{i=1}^{3} \frac{\partial \hat{\Omega}}{\partial \chi_{i}} \chi_{i}-\alpha \frac{\partial \hat{\Omega}}{\partial \alpha}\right]
$$


Thus the equation $\operatorname{curl} \boldsymbol{H}=\mathbf{0}$ reduces to

$$
r H_{\theta}=c_{o}
$$

where $c_{o}$ is a constant.

Regarding the boundary or continuity conditions for a very long tube (in comparison with the exterior radius), we assume they must be satisfied only on the surfaces $r=r_{\mathrm{i}}$ and $r=r_{\mathrm{o}}$. Regarding $(11)_{1}$, considering the expression for $\boldsymbol{B}$ there would be no restriction on the magnetic induction outside, but from $(11)_{1}$, taking into account the expression for $\boldsymbol{H}$, we have that $H_{\theta}^{\mathrm{o}}=H_{\theta}$ and $H_{z}^{\mathrm{o}}=0$ at $r=r_{\mathrm{i}}$ and $r=r_{\mathrm{o}}$, where $\boldsymbol{H}^{\mathrm{o}}$ is the magnetic field outside the tube in vacuum. Therefore from (4) $B_{\theta}^{\mathrm{o}}=\frac{1}{\mu_{0}} H_{\theta}^{\mathrm{o}}$ and $B_{z}^{\mathrm{o}}=0$ (where $\boldsymbol{B}^{\mathrm{o}}$ is the magnetic field outside the tube in vacuum), and then from (12) we have

$$
\tau_{M_{r r}}=\tau_{M_{z z}}=-\frac{1}{2 \mu_{0}} H_{\theta}^{2}, \quad \tau_{\theta \theta}=\frac{1}{2 \mu_{0}} H_{\theta}^{2},
$$

where $H_{\theta}$ would be evaluated at $r=r_{\mathrm{i}}$ and $r=r_{\mathrm{o}}$. From $(11)_{3}$, assuming that on the surface $r=r_{\mathrm{i}}$ there is an external pressure $P_{\mathrm{i}}$ applied, considering the previous results and (77) we obtain

$$
\tau_{r r_{i}}=-P_{\mathrm{i}}-\frac{1}{2 \mu_{0}}\left[H_{\theta}\left(r_{\mathrm{i}}\right)\right]^{2},
$$

while if on the surface $r=r_{\mathrm{o}}$ we assume there is no mechanical load, then considering the last result we obtain a general nonlinear algebraic equation

$$
\int_{r_{\mathrm{i}}}^{r_{\mathrm{o}}} \lambda(\xi) \frac{\partial \tilde{\Omega}}{\partial \lambda}(\xi) \mathrm{d} \xi-P_{\mathrm{i}}-\frac{1}{2 \mu_{0}}\left[H_{\theta}\left(r_{\mathrm{i}}\right)\right]^{2}=-\frac{1}{2 \mu_{0}}\left[H_{\theta}\left(r_{\mathrm{o}}\right)\right]^{2} .
$$

The algebraic equations (81) and (84) can be used to find $r_{\mathrm{i}}$ and $B=B(r)$, where $c_{o}$ would be a given parameter.

Case $\alpha=\pi / 2:$ In that case $\boldsymbol{a}=\boldsymbol{e}_{z}$ and so from (34) we have $H_{\theta}=0$ and

$$
H_{z}=\frac{\partial \hat{\Omega}}{\partial B}+\frac{2}{B}\left[\frac{\partial \hat{\Omega}}{\partial \zeta_{3}}-\sum_{i=1}^{3} \frac{\partial \hat{\Omega}}{\partial \zeta_{i}} \zeta_{i}-\sum_{i=1}^{3} \frac{\partial \hat{\Omega}}{\partial \chi_{i}} \chi_{i}-\alpha \frac{\partial \hat{\Omega}}{\partial \alpha}\right]+\frac{2}{B}\left[\frac{\partial \hat{\Omega}}{\partial \chi_{3}}+\frac{\partial \hat{\Omega}}{\partial \alpha}\right]
$$

Then (2) is satisfied if

$$
H_{z}=c_{o}
$$

where $c_{o}$ is a constant.

Again, using (11) (for brevity we do not show the details here), we can show that $H_{z}^{\mathrm{o}}=H_{z}$ on $r=r_{\mathrm{i}}$ and $r=r_{0}$, and so

$$
\tau_{M_{r r}}=\tau_{M_{\theta \theta}}=-\frac{1}{2 \mu_{0}} H_{z}^{2}, \tau_{M_{z z}}=\frac{1}{2 \mu_{0}} H_{z}^{2} .
$$

If it is assumed again that the tube is under pressure inside and free of mechanical loads outside, we obtain

$$
\int_{r_{\mathrm{i}}}^{r_{\mathrm{o}}} \lambda(\xi) \frac{\partial \tilde{\Omega}}{\partial \lambda}(\xi) \mathrm{d} \xi-P_{\mathrm{i}}-\frac{1}{2 \mu_{0}}\left[H_{z}\left(r_{\mathrm{i}}\right)\right]^{2}=-\frac{1}{2 \mu_{0}}\left[H_{z}\left(r_{\mathrm{o}}\right)\right]^{2},
$$

and this equation along with (86) can be used to find $B=B(r)$ and $r_{\mathrm{i}}$.

\section{Complementary total energy function}

In the above formulation, we use $\boldsymbol{B}_{l}$ as the independent magnetic variable and the dependent variable $\boldsymbol{H}_{l}$ is obtained from $(7)_{2}$. However, treating $\boldsymbol{H}_{l}$ as the independent variable instead of $\boldsymbol{B}_{l}$ has certain advantages, especially in the formulation of variational principles and tackling some boundary value problems [8]. Hence, 
it is convenient to introduce the complementary total energy function $\Omega^{*}$ via a partial Legendre transformation [2]

$$
\Omega^{*}\left(\boldsymbol{C}, \boldsymbol{H}_{l}, \boldsymbol{d}\right)=W\left(\boldsymbol{C}, \boldsymbol{B}_{l}, \boldsymbol{d}\right)-\boldsymbol{H}_{l} \bullet \boldsymbol{B}_{l} .
$$

For this formulation, it is assumed that for every $\boldsymbol{C}$ and $\boldsymbol{d}$ there is a one-to-one relationship between $\boldsymbol{H}_{l}$ and $\boldsymbol{B}_{l}$. The total Cauchy stress for an incompressible solid then takes the form

$$
\boldsymbol{\tau}=2 \boldsymbol{F} \frac{\partial \Omega^{*}}{\partial \boldsymbol{C}} \boldsymbol{F}^{T}-p^{*} \boldsymbol{I}
$$

where $p^{*}$ is the associated Lagrange multiplier due to the incompressibility constraint. From (89) we then have

$$
\boldsymbol{B}_{l}=-\frac{\partial \Omega^{*}}{\partial \boldsymbol{H}_{l}}
$$

For the formulation in this section we set

$$
\boldsymbol{a}=\frac{\boldsymbol{H}_{l}}{H}, \quad H \neq 0,
$$

where $H=\left\|\boldsymbol{H}_{l}\right\|$. Then, in terms of the novel spectral invariants, the complementary total energy function is of the form

$$
\Omega^{*}=\widehat{\Omega}^{*}\left(\lambda_{1}, \lambda_{2}, \lambda_{3}, \zeta_{1}, \zeta_{2}, \zeta_{3}, \xi_{1}, \xi_{2}, \xi_{3}, \chi_{1}, \chi_{2}, \chi_{3}, \alpha, H\right)
$$

The rest of the formulations are similar to the formulations where $\boldsymbol{B}_{l}$ is treated as an independent variable, and hence we omit them in this section.

\section{Further remarks}

If the total energy function is written explicitly in terms of the classical (principal or basic) invariants we can also express it explicitly in terms of the novel spectral invariants in view of (24). Hence, transversely isotropic MS problems can be formulated using either classical or spectral invariants. However, explicit expressions for spectral invariants in terms of classical invariants are not straightforward, especially when two or three of the principal stretches have the same value [57]. Hence, from the onset, we write the total energy function in terms of the novel spectral invariants. Writing the total energy function in terms of spectral invariants is more general (this is evident in dealing with non MS purely isotropic materials, see [61]) than writing in terms of classical invariants.

In the past, when a two-fiber material has been modeled, due to an unclear physical meaning of most of the classical invariants, and the complexity in including all such invariants in the formulations, only a subset of the full set of classical invariants were included in the total strain energy function. However, the authors are convinced that several of these subsets can not fully characterize a two-fiber material. Discussions on which classical invariants should or should not be included in the strain energy function is ongoing and the outcome is not yet clear. In addition to this, a strain energy function written in terms of the classical invariants is not experimentally attractive.

For example, an isochoric uniaxial stretch in one of the preferred directions will perturb the invariants $I_{1}, I_{2}$, $I_{4}, I_{5}, I_{6}, I_{7}, I_{8}$, and $I_{10}$ which is not ideal in obtaining a specific form of strain energy function if the specific form is determined by doing tests that vary one invariant and hold the rest of the invariants constant. However, using spectral invariants, a triaxial test of an incompressible solid, the principal stretches $\lambda_{1}$ and $\lambda_{2}$ can be varied independently. The invariants $\zeta_{1}$ and $\zeta_{2}$ can be varied independently, by applying $\boldsymbol{a}$ in different directions. The invariants $\xi_{1}$ and $\xi_{2}$ can be varied independently by taking different samples, of the same material, with different preferred directions (relative to a principal direction,say). The invariants $\chi_{1}, \chi_{2}$, and $\boldsymbol{a}$ can also be varied independently by applying $\boldsymbol{a}$ in different directions and by taking different samples of the same material with different preferred directions. Obviously, $B$ can be varied easily while keeping the rest of the invariants constant. Hence, it allows us to determine the functional form of $\Omega$ by doing tests that vary one invariant and hold the rest of the invariants constant so that the dependence of $\Omega$ on the remaining invariant can be identified. 
We note in passing that the invariants $I_{1}, I_{2}, I_{4}, I_{5}, I_{6}, I_{7}, I_{8}$, and $I_{10}$ cannot be varied independently in a triaxial test.

Because of their immediate physical meaning it is clear that we should include all the spectral invariants in the total energy function; we note that the classical invariants $I_{4}, I_{6}$, and $I_{8}$ together depend on all the spectral invariants. By writing the total energy function in terms of the spectral invariants directly we avoid its implicit spectral representation via the restricted forms of classical principal invariants. The authors believe that these restricted forms maybe one of the reasons that there is no clear and general methodology on how to choose which classical invariants are suitable. Since all the spectral invariants in the total energy function have to be included, discussions on which invariants should or should not be included in the energy function, as in the case of the classical invariants, are generally not important.

A total energy function for a transversely isotropic MS material written directly in terms of the spectral invariants did not previously exist in the literature. Below, we give an example of a total energy function which is not possible to express explicitly in terms of classical invariants, i.e.

$$
\Omega=\sum_{i=1}^{3} r\left(\lambda_{i}, \zeta_{i}, \xi_{i}, \chi_{i}, H\right),
$$

where

$$
r\left(\lambda_{i}, \zeta_{i}, \xi_{i}, \chi_{i}, H\right)=r_{0}\left(\lambda_{i}\right)+\zeta_{i} r_{1}\left(\lambda_{i}, H\right)+\xi_{i} r_{2}\left(\lambda_{i}\right)+\chi_{i} r_{3}\left(\lambda_{i}, H\right) .
$$

It is clear from the above that the total energy function $\Omega$ has a unique value if two or more of the principal stretches have the same value. We note that in (95) $r_{0}$ and $r_{2}$ are single valued functions of a principal stretch (which has an immediate physical meaning), which facilitates the construction of a specific form of $\Omega$. In the case of $r_{1}$ and $r_{3}$, they only contain two invariants, where an experiment can be easily designed to vary the two invariants independently. Also, since (95) is separable in form, the contribution of each term can be easily analyzed.

At this stage we do not try to fit, for example, the function $r$ to actual experimental data because there is still little usable data in the literature. We can mention, for example, the results for a uniaxial tension test found in [22] and the recent results presented in [60] and [29, 43-45, 64, 65]. Although in [22] there are several curves for the stress versus the stretch for different external axial magnetic fields, it is not mentioned which stress was measured and used for the plots (considering the physical significance, it is theorized that it was most probably the total stress). As well as this, the graphical quality of the plots is not good enough to attempt to fit such data in a good manner. Regarding the experimental results presented in [60], they were obtained using a different formulation, where $\boldsymbol{M}$ (the magnetization) is the independent magnetic variable, therefore in order to use such data some non-trivial conversions are needed.

In this contribution, we have formulated a novel set of spectral invariants for the study of transversely isotropic magneto-mechanically coupled problems. The invariants used in our constitutive equation have an immediate physical interpretation which can easily facilitate experiments to obtain specific forms of the total strain energy. The new set of invariants imparts an experimental advantage over classical invariants presented in the literature, e.g. a simple triaxial test can vary a single invariant while keeping the remaining invariants fixed. Moreover, a total strain energy function, expressed in terms of these invariants, has a symmetry property similar to that of an isotropic elastic solid written in terms of principal stretches.

Transversely isotropic materials are produced when a magnetic field or magnetic induction is applied on the elastomeric particle compound during the curing process. Within this framework of constitutive modeling, such a solid is treated as an anisotropic body with two preferred directions, cf. Shariff and Bustamante [59] and Shariff [57]. Under the application of a magnetic load, the particles align in a preferred direction and another preferred direction is created by the externally applied magnetic field.

In contrast to magneto-mechanically coupled models based on classical invariants, various expressions for the total stresses in terms of the deformation and the magnetic induction resulting from spectral invariants are simpler when considering different classical homogeneous and non-homogeneous problems, e.g. triaxial extension of a slab, extension and simple torsion of a circular cylinder, and inflation and extension of a tube. Since elastomeric materials show typical viscoelastic behavior, an extension to the viscoelastic rate-dependent case is going to be dealt with in another contribution. There are plans to implement the model in a magnetomechanical coupled finite element framework in order to perform simulations of more complex boundary value problems. 


\section{Declaration of Conflicting Interests}

The author(s) declared no potential conflicts of interest with respect to the research, authorship, and/or publication of this article.

\section{Funding}

The third and fourth authors acknowledge the funding by an ERC advanced grant within the project MOCOPOLY.

\section{Note}

1. For simplicity and brevity we consider $\boldsymbol{B}_{l}$ as the independent magnetic variable, but it is possible to reformulate the theory considering instead $\boldsymbol{H}_{l}$ as the independent magnetic variable, see $\S 3.7$ of [9] and Section 5 of the present work.

\section{References}

[1] Brigadnov, IA and Dorfmann, A. Mathematical modeling of magneto-sensitive elastomers. Int J Solids Struct 2003; 40: 46594674.

[2] Bustamante, R. Transversely isotropic nonlinear magneto-active elastomers. Acta Mech 2010; 210 : $183-214$.

[3] Bustamante, R, Dorfmann, A and Ogden, RW. On variational formulations in nonlinear magnetoelastostatics. Math Mech Solids 2008; 13: 725-745.

[4] Bustamante, R, Dorfmann, A and Ogden, RW. Numerical solution of finite geometry boundary value problems in nonlinear magnetoelasticity. Int $J$ Solids Struct 2011; 48: 874-883.

[5] Chatzigeorgiou, G, Javili, A and Steinmann, P. Unified magnetomechanical homogenization framework with application to magnetorheological elastomers. Math Mech Solids 2014; 19: 193-211.

[6] Danas, K, Kankanala, SV and Triantafyllidis, N. Experiments and modelling of iron-particled-filled magnetorheological elastomers. J Mech Phys Solids 2012; 60(1): 120-138.

[7] Dorfmann, A and Ogden, RW. Magnetoelastic modelling of elastomers. Eur J Mech A-Solids 2003; 22: $497-507$.

[8] Dorfmann, A and Ogden, RW. Nonlinear magnetoelastic deformations of elastomers. Acta Mech 2004a; 167: 13-28.

[9] Dorfmann, A and Ogden, RW. Nonlinear magnetoelastic deformations. Q J Mech Appl Math 2004b; 57: 599-622.

[10] Dorfmann, A and Ogden, RW. Some problems in nonlinear magnetoelasticity. Z Angew Math Phys (ZAMP) $2005 ; 56: 718-745$.

[11] Galipeau, E and Ponte-Castañeda, P. Giant field-induced strains in magnetoactive elastomers composites. Proc R Soc 2013; 469: 20130385.

[12] Kankanala, SV and Triantafyllidis, N. On finitely strained magnetorheological elastomers. J Mech Phys Solids 2004; 52: 28692908.

[13] Ogden, RW and Steigmann, DJ. Mechanics and electrodynamics of magneto- and electro-elastic materials (CISM Courses And Lectures Series, vol. 527). Wien: Springer, 2010.

[14] Ponte-Castañeda, P and Galipeau, E. Homogenization-based constitutive models for magnetorheological elastomers at finite strain. J Mech Phys Solids 2011; 59: 194-215.

[15] Pucci, E and Saccomandi, G. On the controllable states of elastic dielectric and magnetoelastic solids. Int J Eng Sci 1993; 31: 251-256.

[16] Salas, E and Bustamante, R. Numerical solution of some boundary value problems in nonlinear magneto-elasticity. $J$ Intel Mat Syst Str 2015; 26(2): 156-171.

[17] Saxena, P, Hossain, H and Steinmann, P. Nonlinear magneto-viscoelasticity of transversally isotropic magneto-active polymers. Proc R Soc A 2014; 470: 20140082.

[18] Saxena, P, Hossain, H and Steinmann, P. A theory of finite deformation magneto-viscoelasticity. Int J Solids Struct 2014; 50: 3886-3897.

[19] Steigmann, DJ. Equilibrium theory for magnetic elastomers and magnetoelastic membranes. Int J Nonlinear Mech 2004; 39: 1193-1216.

[20] Vu, DK and Steinmann, P. Material and spatial motion problems in nonlinear electro- and magneto-elastostatics. Math Mech Solids 2010; 15: 239-257.

[21] Bednarek, S. The giant magnetostriction in ferromagnetic composites within an elastomer matrix. Appl Phys A-Mater 1999; 68: 63-67.

[22] Bellan, C and Bossis, G. Field dependence of viscoelastic properties of MR elastomers. Int J Mod Phys B 2002; 16: 2447-2453.

[23] Bica, I. The influence of the magnetic field on the elastic properties of anisotropic magnetorheological elastomers. J Ind Eng Chem 2012; 18: 1666-1669.

[24] Boczkowska, A and Awietjan, SF. Smart composites of urethane elastomers with carbonyl iron. J Mater Sci 2009; 44: 4104-4111.

[25] Boczkowska, A and Awietjan, SF. Microstructure and properties of magnetorheological elastomers. In: Boczkowska, A (ed.) Advanced elastomers-technology, properties and applications. Croatia: InTech, DOI: 10.5772/2784, 2012, 147-180.

[26] Böse, H. Viscoelastic properties of silicone-based magnetorheological elastomers. Int J Mod Phys B 2007; 21: $4790-4797$. 
[27] Deng, H and Gong, X. Application of magnetorheological elastomer to vibration absorber. Commun Nonlinear Sci 2008; 13: 1938-1947.

[28] Ghafoorianfar, N, Wang, X and Gordaninejad, F. On the sensing of magnetorheological elastomers. In: Lynch, JP et al. (eds.) Sensors and smart structures technologies for civil, mechanical, and aerospace systems (SPIE Proceedings, vol. 8692). SPIE Press, 2013, paper 869214.

[29] Ginder, JM, Clark, SM, Schlotter, WF and Nichols, ME. Magnetostrictive phenomena in magnetorheological elastomers. Int J Mod Phys B 2002; 16: 2412-2418.

[30] Gordaninejad, F, Wang, W and Mysore, P. Behavior of thick magnetorheological elastomers. J Intel Mat Syst Str 2012; 23: 1033-1039.

[31] Jolly, MR, Carlson, JD and Muñoz, BC. A model of the behaviour of magnetorheological materials. Smart Mater Struct 1996; 5: 607-614.

[32] Lokander, M and Stenberg, B. Performance of isotropic magnetorheological rubber materials. Polym Test 2003; 22: $245-251$.

[33] Mitsumata, T. Recent progress in magnetorheological gels and elastomers. Recent Pat Chem Eng 2009; 2: $159-166$.

[34] Mitsumata, T and Ohori, S. Magnetic polyurethane elastomers with wide range modulation of elasticity. Polym Chem 2011; 2: 1063-1067.

[35] Varga, Z, Filipcsei, G and Zrínyi, M. Magnetic field sensitive functional elastomers with tuneable modulus. Polymer 2006; 47: 227-233.

[36] Brown, WF. Magnetoelastic Interactions. Berlin: Springer, 1966.

[37] Eringen, AC and Maugin, GA. Electrodynamics of Continua I. New York: Springer, 1990.

[38] Maugin, GA. Continuum Mechanics of Electromagnetic Solids. Amsterdam: North Holland, 1988.

[39] Hutter, K, van de Ven, AAF and Ursescu, A. Electromagnetic Field Matter Interactions in Thermoelastic Solids and Viscous Fluids. Berlin: Springer, 2006.

[40] Albanese, AM and Cunefare, KA. Properties of magnetorheological semiactive vibration absorber. In Agnes, GS and Wang, K-W (eds.) Smart structures and materials: Damping and isolation (SPIE Proceedings, vol. 5052). SPIE Press, $2003,36-43$.

[41] Böse, H, Rabindranath, R and Ehrlich, J. Soft magnetorheological elastomers as new actuators for valves. J Intel Mat Syst Str 2012; 23: 989-994.

[42] Farshad, M and Le Roux, M. A new active noise abatement barrier system. Polym Test 2004; 23: 855-860.

[43] Ginder, JM, Nichols, ME, Elie, LD and Tardiff, JL. Magnetorheological elastomers: properties and applications. In: Wuttig, MR (ed.) Smart structures and materials: smart materials technologies (SPIE Proceedings, vol. 3675). SPIE Press, $1999,131-138$.

[44] Ginder, JM, Nichols, ME, Elie, LD and Clark, SM. Controllable stiffness components based on magnetorheological elastomers. In: Wereley, NM (ed.) Smart structures and materials: smart structures and integrated systems (SPIE Proceedings, vol. 3985). SPIE Press, 2000, 418-425.

[45] Ginder, JM, Schlotter, WF and Nichols, ME. Magnetorheological elastomers in tunable vibration absorbers. In: Inman, DJ (ed.) Smart structures and materials: damping and isolation (SPIE Proceedings vol. 4331). SPIE Press, 2001, 103-110.

[46] Kashima, S, Miyasaka, F and Hirata, K. Novel soft actuator using magnetorheological elastomer. IEEE T Magn 2012; 48: 16491652.

[47] Li, W and Zhang, X. Research and applications of MR elastomers. Recent Pat Mech Eng 2008; 1: 161-166.

[48] Yalcintas, M and Dai, H. Vibration suppression capabilities of magnetorheological materials based adaptive structures. Smart Mater Struct 2004; 13: 1-11.

[49] Zhu, JT, Xu, ZD and Guo, YQ. Magnetoviscoelasticity parametric model of a MR elastomer vibration device. Smart Mater Struct 2012; 21: 075034.

[50] Varga, Z, Filipcsei, G and Zríngi, M. Smart composites with controlled anisotropy. Polymer 2005; 47: $7779-7787$.

[51] Hossain, M, Saxena, P and Steinmann, P. Modelling the mechanical aspects of the curing process of magneto-sensitive elastomeric materials. Int J Solids Struct 2015; 58: 257-269.

[52] Hossain, M, Chatzigeorgiou, G, Meraghni, F and Steinmann, P. A multi-scale approach to model the curing process in magnetosensitive polymeric materials. Int J Solids Struct 2015; 69-70: 34-44.

[53] Bustamante, R and Merodio, J. Constitutive structure in coupled non-linear electro-elasticity: invariants descriptions and constitutive restrictions. Int J Nonlinear Mech 2011; 46: 1315-1323.

[54] Spencer, AJM. Theory of invariants. In: Eringen, AC (ed.) Continuum physics, vol. 1. New York: Academic Press, 1971, $239-353$.

[55] Zheng, QS. Theory of representations for tensor functions. A unified invariant approach to constitutive equations. Appl Mech Rev 1994; 47: 545-587.

[56] Shariff, MHBM. Nonlinear transversely isotropic elastic solids: an alternative representation. QJ Mech Appl Math 2008; 61: 129-149.

[57] Shariff, MHBM. Physical invariants for nonlinear orthotropic solids. Int J Solids Struct 2011; 48: $1906-1914$.

[58] Shariff, MHBM. Nonlinear orthotropic elasticity: only six invariants are independent. J Elasticity 2013; 110: $237-241$.

[59] Shariff, MHBM and Bustamante, R. On the independence of strain invariants of two preferred direction nonlinear elasticity. Int J Eng Sci 2015; 97: 18-25.

[60] Bustamante, R and Shariff, MHBM. A principal axis formulation for nonlinear magnetoelastic deformations: isotropic bodies. Eur J Mech A-Solids 2015; 50: 17-27.

[61] Ogden, RW. Non-linear Elastic Deformations. New York: Dover, 1997. 
[62] Truesdell, CA and Toupin, R. The classical field theories. In: Handbuch der physik, vol. III/1. Berlin: Springer, 1960.

[63] Kovetz, A. Electromagnetic Theory Oxford: University Press, 2000.

[64] Hossain, M and Steinmann, P. More hyperelastic models for rubber-like materials: consistent tangent operator and comparative study. J Mech Behav Mater 2013; 22(1-2): 27-50.

[65] Tiersten, HF. Coupled magnetomechanical equations for magnetically saturated insulators. J Math Phys 1964; 5: $1298-1318$.

\section{Appendix A: Uniaxial deformation using $\Omega^{*}$}

The homogeneous deformation of an incompressible circular cylindrical bar is described by

$$
r=\lambda^{-\frac{1}{2}} R, \quad \theta=\Theta, \quad z=\lambda Z,
$$

where $(R, \Theta, Z)$ are cylindrical polar coordinates in the reference configuration and $(r, \theta, z)$ the corresponding coordinates in the deformed configuration and $\lambda>0$ is the axial stretch. This type of deformation was used by Bellan and Bossis [22] to obtain the experimental results. In this case $\boldsymbol{F} \equiv \operatorname{diag}\left(\lambda^{-\frac{1}{2}}, \lambda^{-\frac{1}{2}}, \lambda\right)$. For the Bellan and Bossis [22] experiment $\boldsymbol{a}=\boldsymbol{d} \equiv[0,0,1]^{\mathrm{T}} \cdot \boldsymbol{H}_{l}=H \boldsymbol{a} \equiv(0,0, H)^{\mathrm{T}}$ and hence $\boldsymbol{H} \equiv\left(0,0, \lambda^{-1} H\right)^{\mathrm{T}}, \zeta_{1-2}=$ $\xi_{1-2}=\chi_{1-2}=0$ and $\alpha=\zeta_{3}=\xi_{3}=\chi_{3}=1$. In view of (91), (3.2) and (5), we have

$$
\boldsymbol{B}_{l}=-\frac{\partial \Omega^{*}}{\partial H} \boldsymbol{a}, \quad \boldsymbol{B}=-\lambda \frac{\partial \Omega^{*}}{\partial H} \boldsymbol{a}, \quad \boldsymbol{M}=M \boldsymbol{a}=-\frac{1}{\mu_{0}}\left[\lambda \frac{\partial \Omega^{*}}{\partial H}+\mu_{0} \frac{H}{\lambda}\right] \boldsymbol{a} .
$$

For the special form of the constitutive equation described previously, the axial stress is given by

$$
\tau_{z z}=\lambda r^{\prime}(\lambda, 1,1,1, H)-\lambda^{-\frac{1}{2}} r^{\prime}\left(\lambda^{-\frac{1}{2}}, 0,0,0, H\right)-\frac{\mu_{0} H^{2}}{2 \lambda^{2}} .
$$

The derivation of the above equation takes the Maxwell stress into account exterior to the cylinder

$$
\tau_{M_{r r}}=\tau_{M_{\theta \theta}}=-\tau_{M_{z z}}=-\frac{\mu_{0} H^{2}}{2 \lambda^{2}}
$$

